\title{
Optimal timing of type A intramural hematoma repair
}

\author{
Zain Al Rstum, Akiko Tanaka, Steven B. Eisenberg, Anthony L. Estrera \\ Department of Cardiothoracic and Vascular Surgery, McGovern Medical School at The University of Texas Health Science Center at Houston \\ (UTHealth), Houston, TX, USA \\ Correspondence to: Anthony L. Estrera, MD, FACS. Professor and Chief of Cardiac Surgery, Department of Cardiothoracic and Vascular Surgery, \\ McGovern Medical School at UTHealth, 6400 Fannin St., Suite 2850, Houston, TX 77030, USA. Email: Anthony.L.Estrera@uth.tmc.edu.
}

\begin{abstract}
Acute type A aortic intramural hematomas (IMHs) are often included under the spectrum of acute aortic syndromes. The classical definition is the presence of hematoma in the media without identifiable intimal tear. Dissection occurring within two weeks of presentation is defined as acute. Acute type A IMH remains a subject of debate, especially regarding its definition and management. The classical theory of pathogenesis of IMHs is ruptured vasa vasorum in the aortic media. However, the majority of IMHs are now detected with an intimal defect using high-resolution computed tomography and intravascular ultrasound, which implies that IMHs may be a subset of aortic dissections (ADs), with very limited flow in the false lumen. Much controversy remains regarding IMH differences in presentation, diagnosis, and risk for progression. Geographic location and ethnicity, especially Asian vs. Western, possibly affect the natural history and outcomes of acute type A IMH. In this review, we describe the pathophysiology and management strategies for acute type A IMHs.
\end{abstract}

Keywords: Intramural hematoma (IMH); penetrating aortic ulcer (PAU); aortic dissection (AD)

Submitted Mar 25, 2019. Accepted for publication: Jul 18, 2019.

doi: $10.21037 /$ acs.2019.07.08

View this article at: http://dx.doi.org/10.21037/acs.2019.07.08

\section{Introduction}

Intramural hematoma (IMH) was first described by Krukenberg in 1920 during postmortem examination, in which he found a "dissection without intimal tear" (1). This was later adopted and widely accepted as the original definition of IMH. The definition later evolved into a hematoma confined within the medial layer of the aorta in the absence of a detectable intimal tear. Some believe that $\mathrm{IMH}$ represents acute aortic dissection $(\mathrm{AD})$ with thrombosis of the false lumen and that an intimal tear is always present, whether or not it is identified (1). This conclusion was driven by some reports that showed presence of intimal defects in approximately $70 \%$ of initially diagnosed IMHs using highresolution computed tomography $(\mathrm{CT})(1,2)$ and at the time of surgery (1-3). Thus, the very existence of IMH itself is now questioned (1-4).

\section{Incidence and pathophysiology}

IMH accounts for $5-18 \%$ of acute aortic syndromes (2). In a review of 1,010 patients in the International Registry of Acute Aortic Dissection (IRAD), IMH was present in 58 patients $(5.7 \%)$. Among 51 patients with only aortic IMH at the initial diagnosis, eight (16 percent) progressed to $\mathrm{AD}$ on serial imaging studies (2). Acute aortic syndrome is a term used to describe a constellation of life-threatening aortic diseases that have similar presentation, but also have distinct demographic, clinical, pathological and survival characteristics $(2,3)$. Many classify acute aortic syndrome into three major entities: $\mathrm{AD}, \mathrm{IMH}$ and penetrating aortic ulcer (PAU). These make up a spectrum of aortic disease, in which one entity may evolve into or coexist with another (3).

IMH has been described primarily as a disease of the aortic media, and is distinguished from classical $\mathrm{AD}$, which is a combination of medial and intimal diseases $(3,4)$. There are several theories that exist regarding the mechanism of IMH development, without strong evidence to support any one theory. Debate exists over whether IMHs are the result of ruptured vasa vasorum or thrombosed false lumen $\mathrm{AD}$ with undetected intimal tear or a combination thereof. The classical theory, although lacking in robust 


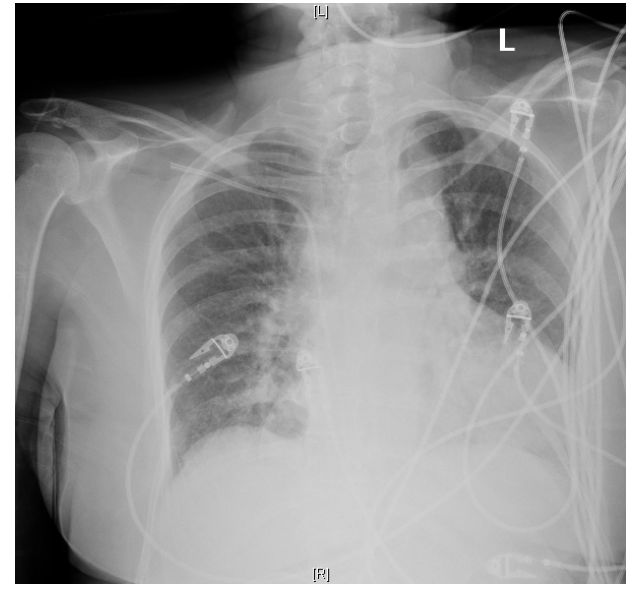

Figure 1 Widened mediastinum in a patient with type A intramural hematoma (IMH).

evidence, suggests that IMH is caused by rupture of the vasa vasorum in the media. This rupture subsequently leads to seperation of the medial wall layers, eventually causing a secondary tear (i.e., intimal defect) or communication to the adventitial space (4). Again, strong evidence on this theory is lacking and it has been argued that IMH is not solely a disease of vasa vasorum in the media, but is instead a disease of intima as well. This is supported by reported cases of iatrogenic IMH after instrumentation of the aorta for left heart catheterization or other procedures (5-7). In addition, some studies demonstrate IMHs have a small intimal communication discovered at the time of surgery (2,4,7-10) or, more recently, with high-resolution CT. Studies examining the vasa vasorum have suggested that hyperplasia leads to chronic occlusive disease within the aortic wall, which triggers chronic medial ischemia and degeneration (8). Notably, this medial ischemia often causes hematoma and dissection in the outer third of the media $(2,9,10)$. Further studies are needed to improve understanding of the pathophysiology of IMH.

\section{Presentation and clinical features}

Patients with acute type A IMH can present with variety of symptoms, with acute onset of severe chest or back pain being most common, occurring in $80 \%$ to $90 \%$ (10). Although painless dissection with IMH has been reported, it is relatively uncommon (11). The pain is usually described as severe, sharp or knife-like, categorically unlike any pain experienced before, and causes the patient to seek medical attention within minutes to hours of onset. Acute type A IMH can also be associated with shortness of breath. Symptoms that denote presence of organ malperfusion may be present, especially with descending thoracic aortic involvement. Cerebral, spinal, visceral, renal, and limb malperfusions can clinically present as syncope, stroke, paralysis, abdominal pain, renal dysfunction, or limb pain. Although clinical presentations may be very similar to acute type A ADs, there are differences. As demonstrated in our previous study, patients with IMH present less frequently with shortness of breath, malperfusion syndromes, significant aortic insufficiency, hypotension, and rupture compared to classical AD (10). Several other studies have reported a higher risk of rupture with $\mathrm{IMH}$ as compared to $\mathrm{AD}(12,13)$. Patients with $\mathrm{IMH}$ are usually older, female dominant $(4,10)$ more commonly present with aortic aneurysm, and few have Marfan syndrome $(1,10,12,14)$.

\section{Diagnosis}

The diagnosis of acute type A IMH and other acute aortic syndrome entities relies on clinical presentation and imaging. No reliable biomarkers are available to provide an accurate diagnosis of any acute aortic syndromes. Electrocardiogram is usually obtained as part of chest pain work up to rule out coronary artery ischemia. Plain chest films may show a widened mediastinum (Figure 1), which raises suspicion for aortic disease, however, plain $\mathrm{X}$-rays are not a sensitive diagnostic investigation for IMH or acute aortic syndromes $(14,15)$. CT angiography (CTA) is the diagnostic imaging modality of choice in hemodynamically stable patients. It is highly sensitive and specific for identifying aortic pathology (Figure 2). The imaging study should include the entire aorta from the aortic root to the femoral vessels. We often receive images sent together with patient transfers that mostly include pulmonary embolism protocol CTA chest. In such images, evaluation of the aorta is limited to the chest, and timing of the contrast may also be inadequate to visualize the aortic pathology. If CTA is equivocal or the patient is hemodynamically unstable, transesophageal echocardiography (TEE) may be useful to confirm and support the diagnosis. Sensitivity of TEE has been reported as high as $100 \%$, with a specificity of $91 \%$ $(16,17)$. TEE can also identify hemorrhagic pericardial effusion, tamponade, aortic valve regurgitation, and regional wall motion abnormalities. However, complete imaging of the distal arch and descending aorta is limited with TEE and it is highly operator dependent. Thus, TEE may not 

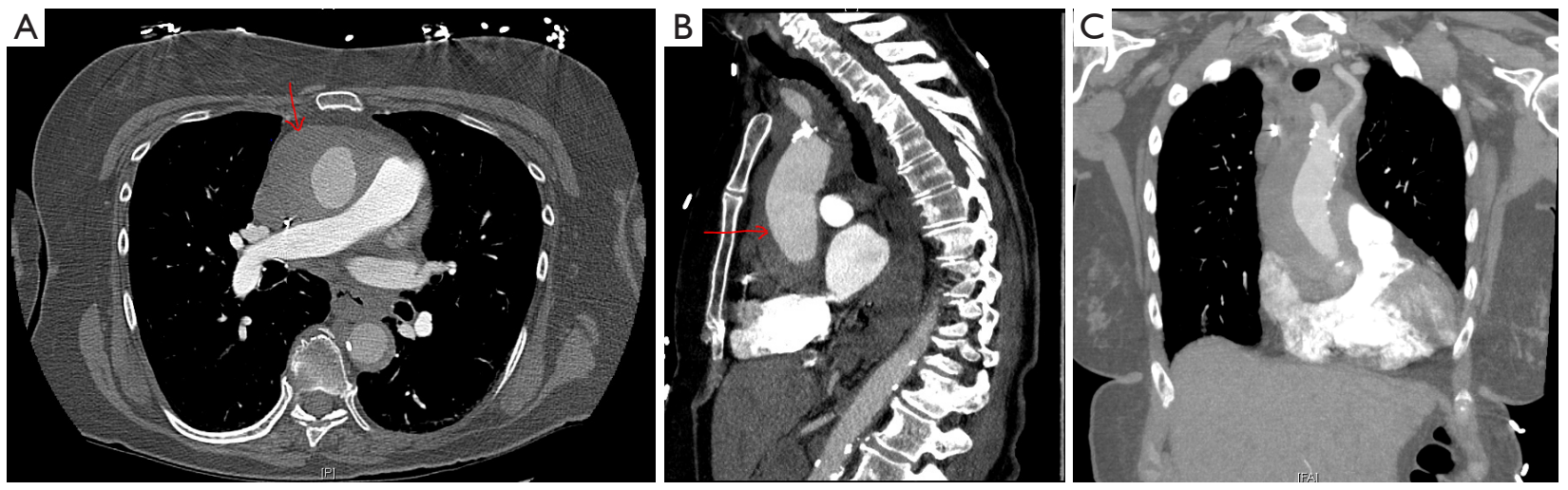

Figure 2 Type A intramural hematoma. (A) Contrast-enhanced axial CT scan shows no enhancement of attenuation in the crescent-shaped areas (arrow); (B) contrast-enhanced sagittal CT scan shows intramural hematoma (arrow); (C) coronal CT scan shows intramural hematoma.

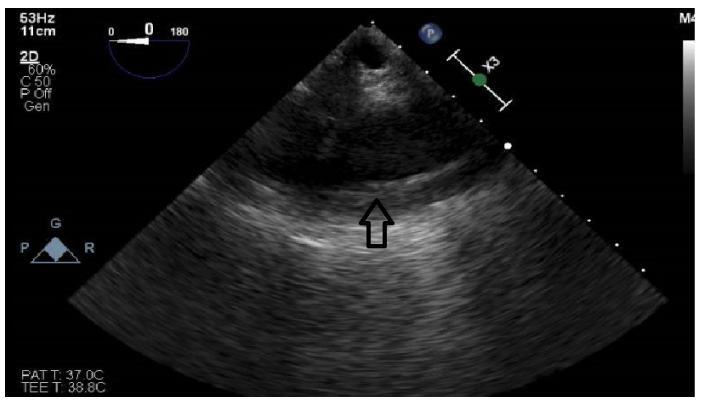

Figure 3 Type A intramural hematoma on transesophageal echocardiogram (TEE) with crescentic thickening of the aortic wall (arrow).

be suitable for assessing the differential diagnoses within the spectrum of aortic syndromes (17). Magnetic resonance imaging is seldom used in the acute setting, due to the wider availability of CT in the emergency department, and due to the longer duration of scanning time for MRI. Aortogram was a gold standard in the past but is rarely necessary today with the advent of CTA $(16,18)$.

The main finding on CTA in IMH is a crescentic or circular high attenuation area along the aortic wall that does not enhance with contrast, absence of an intimal flap, and absence of compression of the patent lumen (18). This is in contrast to classical $\mathrm{AD}$, which radiographically appears with two distinct lumens. However, it is sometimes difficult to distinguish between aortic IMH and an acute dissection with complete thrombosis of the false lumen, as they appear similar. In this scenario, intravascular ultrasound (IVUS) may have a role (19). On TEE, findings include regional thickening of the aortic wall in a crescentic or circular shape and/or evidence of intramural accumulation of blood $(5,6,18)$ (Figure 3).

\section{Management}

A crucial aspect of management of patients with acute $\mathrm{IMH}$ is ensuring an early and correct diagnosis so that the appropriate treatment can be instituted in a timely fashion. We treat acute type A IMH as any other acute aortic syndrome, and its initial management includes pain control and anti-impulse therapy, in the absence of hypotension, by controlling the heart rate and blood pressure to minimize the likelihood of rupture or progression (10). These measures should be initiated immediately in the intensive care unit for all patients once the diagnosis has been established and should not interfere with the timely transfer to the operating room for those with indications for immediate aortic repair. Blood pressure control agents are typically comprised of continuous infusion of beta blockers, such as esmolol or labetalol. Beta blocker infusions are initiated to maintain heart rate $<60 \mathrm{bpm}$ and systolic blood pressure between 100 and $120 \mathrm{mmHg}$, thereby decreasing shear stress and minimizing lesion progression (20). Calcium channel blockers (verapamil or diltiazem) can be used as alternatives or as additional blood pressure control. Vasodilator therapy (e.g., nitroprusside) should not be used without first controlling heart rate with beta blockade since it induces reflex activation of the sympathetic nervous system when used alone, leading to enhanced ventricular contraction and increased aortic shear stress. After initial stabilization of patients, definitive management is sought. The management of acute type A IMH remains 


\begin{tabular}{|c|c|c|}
\hline Major differences & Eastern $^{\# \#}$ & Western" \\
\hline Incidence (in ATAAD) & $17-40 \%$ & $5-19 \%$ \\
\hline Female & $40-70 \%$ & $30-40 \%$ \\
\hline Age & $54-63$ years & $60-65$ years \\
\hline Initial medical & $50-88 \%$ & $0-49 \%$ \\
\hline Mortality surgery & $9-12 \%$ & $21-33 \%$ \\
\hline Mortality medical & $4-8 \%$ & $33-78 \%$ \\
\hline \multicolumn{3}{|c|}{$\begin{array}{l}{ }^{\#} \text {, based on data from most studies reported from North America } \\
\text { and Europe }(10,12,14,15,24-26) ;{ }^{\#} \text {, based on data reported } \\
\text { in studies published by Korean, Japanese, and Chinese } \\
\text { investigators }(8,9,21-23,27,28) \text {. ATAIMH, acute type A intramural } \\
\text { hematoma; ATAAD, acute type A aortic dissection. }\end{array}$} \\
\hline
\end{tabular}

controversial because it appears that pathology, risk of progression, and outcomes appear to be dependent on geographic location and, possibly, on ethnicity. Previous reports have demonstrated obvious differences between Asian and Western cohorts: the Asian series report a greater incidence of type A IMH, and the patients are more frequently women, at greater risk for progression to typical dissection with associated complications, and have a lower mortality when managed medically (10, 21-23).

In contrast, many Western reports maintain that type A IMH acts similarly to typical dissection, and thus recommend a more aggressive stance $(10,12,14,15)$. The reasons for these differences between geographic location or by ethnicity remain unclear, although some have suggested that aggressive radiologic surveillance programs may explain the higher incidence of IMH in Asian cohorts (17). Differences in incidence and treatment outcomes are listed in Table 1 (24).

\section{Surgical approach}

In general, type A aortic pathologies are repaired using open surgical techniques, while an initial endovascular approach is appropriate for most type B aortic pathologies, provided the patient's anatomy is suitable for endograft placement. However, as mentioned, there are different approaches to treat $\mathrm{IMH}$, which is mostly managed medically in Asian cohorts (10,21-23) and managed aggressively with surgical repair similar to typical acute type A dissection in Western cohorts $(10,12,14,15)$. We have adopted a slightly different timing for the surgical approach to type A IMH. In our previous reports $(10,24)$, we examined early outcomes of a selective, delayed (eventual) approach to the treatment of type A IMH. We found that conversions to classic dissections rarely occur within 3 days of onset, therefore a delayed surgical approach was adopted. This approach was based on the hypothesis that a short delay in surgical intervention might allow the inflammation to subside, tissues to become more manageable and thus facilitate subsequent repair (10). We adopted an approach of eventual repair in 2 to 3 days for patients with acute type A IMH who remain stable with no signs of progression and a mean time to repair of 80 hours $(10,24)$. The results of this approach demonstrated no difference in early or late outcomes compared with those obtained by the standard, immediate surgical management of typical type A dissection $(10,24)$. Although delayed surgery conferred no advantages, in recognition of the fact that no early (less than 3 days) rupture occurred when patients were clinically stable, we currently intervene urgently, as opposed to an emergent fashion, for acute type A IMH. It must be emphasized that this delayed approach requires the patient to be hemodynamically and clinically stable with minimal pain, and managed in an intensive care unit so that effective antiimpulse therapy is achieved.

\section{Surgical technique}

We take the same surgical approach to patients with $\mathrm{IMH}$ as we do for acute type A repair (Figure 4). Surgical management includes utilization of cardiopulmonary bypass in a standard fashion, deep hypothermic circulatory arrest, and retrograde cerebral perfusion. Cardiopulmonary bypass is routinely initiated via femoral or axillary artery cannulation. In some cases, we cannulate the ascending aorta with the Seldinger technique and confirm wire position in the true lumen with transesophageal echocardiogram. Axillary artery cannulation is used in cases of femoral artery pulse deficit. Adequate arterial inflow perfusion is confirmed by monitoring cerebral oximetry and appropriate decreases in nasopharyngeal and bladder temperatures. Venous cannulation is via the superior and inferior vena cava. Both antegrade and retrograde cold blood cardioplegia provide myocardial protection. A left ventricular sump graft catheter is inserted into the right superior pulmonary vein. Once adequate cooling is achieved, cardiopulmonary bypass is discontinued and the circulation is arrested. Retrograde cerebral perfusion 


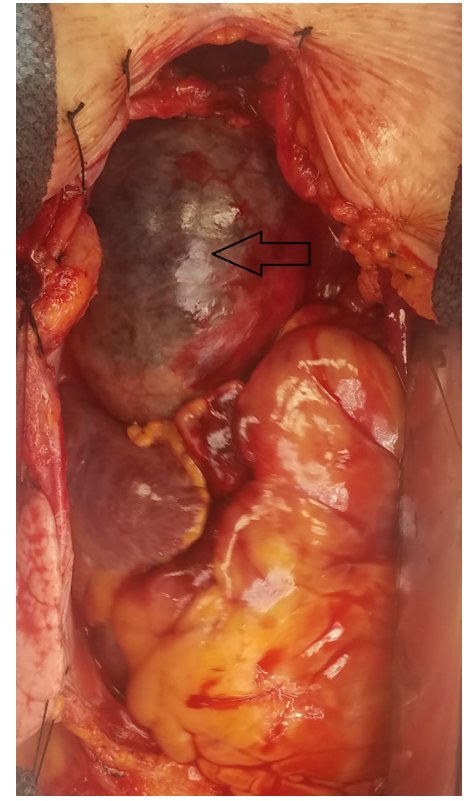

Figure 4 Intraoperative view of the type A intramural hematoma (arrow).

is started via the superior vena cava cannula and the cava is snared. The patient is placed in slight Trendelenburg position. The ascending aorta is then opened and inspected. The arch is replaced only if enlarged $>5 \mathrm{~cm}$; partial arch replacement (hemiarch) with an open distal anastomosis is performed otherwise. Any remaining thrombus within the wall is carefully removed before performing the anastomosis. Once the anastomosis is complete, the graft is desired and clamped, and antegrade perfusion is initiated via a sidearm branch of the aortic graft. After systemic warming has begun, the proximal reconstruction is performed. The remaining ascending aorta is resected to the sinotubular junction. If the aortic valve is intact and the aortic root is not enlarged $>5 \mathrm{~cm}$ or compromised, the proximal anastomosis is performed after resuspension of the aortic valve. Once the anastomosis is accomplished, we rewarm the patient's heart to a myocardial septal temperature of
$30{ }^{\circ} \mathrm{C}$, and then release the ascending aortic clamp and restore the flow to the coronary circulation. Once the patient's core body temperature is $36^{\circ} \mathrm{C}$ and they are able to maintain good systemic blood pressure and sinus rhythm, they are weaned from cardiopulmonary bypass in standard fashion.

\section{Outcomes}

In our experience, overall 30-day mortality after aortic repair was $15.3 \%$, without significant difference seen between acute type A IMH and typical acute type A AD patients $12 \%(12 / 101)$ vs. $16 \%(68 / 422 ; \mathrm{P}=0.289)(10,24)$. This is also true for the unadjusted 10 -year survival between the two groups. However, the difference in effect of acute type A IMH vs. classical acute type A dissection on mortality became apparent and pronounced following adjustment for age and baseline renal function. Long-term survival at 1-, 5- and 10-year for acute type A IMH was $85.8 \%, 81.1 \%$, and $66.7 \%$, respectively, and was significantly better than survival of classic acute type A AD: $78.8 \%, 72.4 \%$, and $54.3 \%$, respectively $(\mathrm{P}<0.039)(10,24)$. Table 2 summarizes postoperative outcomes between type $\mathrm{A} A D$ and $\mathrm{IMH}$ (24).

\section{Conclusions}

Acute type A IMH continues to be associated with significant morbidity and mortality that is comparable to classic AD. Our previously published results show favorable operative outcomes with the institutional approach of deferred (beyond 72 hours) intervention for IMH in all but unstable patients, which are comparable to the outcomes after emergent surgery in acute type A AD. These findings suggest that "timely" intervention (within the first 3 days) can prove beneficial to reduce operative mortality for stable acute type A IMH. A multidisciplinary management approach involving aggressive medical management and risk stratification for timely surgical intervention, along with genetic profiling, is recommended for optimal care. 


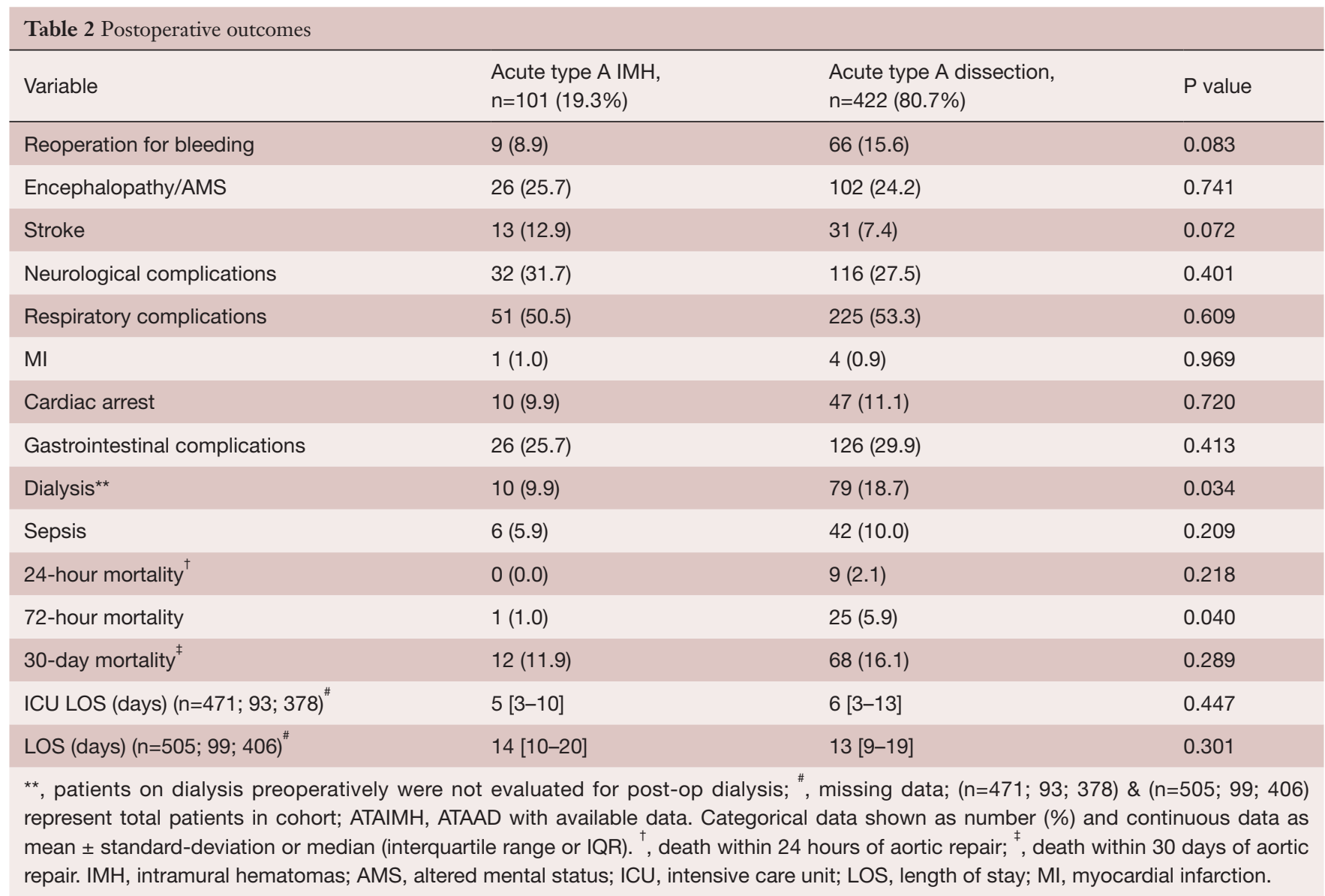

\section{Acknowledgments}

None.

\section{Footnote}

Conflicts of Interest: Dr. Estrera is a consultant for WL Gore. The other authors have no conflicts of interest to declare.

\section{References}

1. Uchida K, Imoto K, Takahashi M, et al. Pathologic characteristics and surgical indications of superacute type A intramural hematoma. Ann Thorac Surg 2005;79:1518-21.

2. Evangelista A, Mukherjee D, Mehta RH, et al. Acute intramural hematoma of the aorta: a mystery in evolution. Circulation 2005;111:1063-70.

3. Corvera JS. Acute aortic syndrome. Ann Cardiothorac Surg 2016;5:188-93.

4. Song JK. Diagnosis of aortic intramural hematoma. Heart
2004;90:368-71.

5. Vilacosta I, Castillo JA, Peral V, et al. Intramural aortic hematoma following intra-aortic balloon counter pulsation. Documentation by transesophageal echocardiography. Eur Heart J 1995;16:2015-6.

6. Vilacosta I, de Dios RM, Pinto AG. Aortic intramural hematoma during coronary angioplasty: insights into the pathogenesis of intramedial hemorrhage. J Am Soc Echocardiogr 2000;13:403-6.

7. Berdat PA, Carrel T. Aortic dissection limited to the ascending aorta mimicking intramural hematoma. Eur J Cardiothorac Surg 1999;15:108-9.

8. Tanaka H, Zaima N, Sasaki T, et al. Adventitial vasa vasorum arteriosclerosis in abdominal aortic aneurysm. PloS One 2013;8:e57398.

9. Osada H, Kyogoku M, Ishidou M, et al. Aortic dissection in the outer third of the media: what is the role of the vasa vasorum in the triggering process?. Eur J Cardiothorac Surg 2013;43:e82-8. 
10. Estrera AL, Sandhu HK, Leake SS, et al. Early and late outcomes of acute type A aortic dissection with intramural hematoma. J Thorac Cardiovasc Surg 2015;149:137-42.

11. Park SW, Hutchison S, Mehta RH, et al. Association of painless acute aortic dissection with increased mortality. Mayo Clin Proc 2004;79:1252-7.

12. Harris KM, Braverman AC, Eagle KA, et al. Acute aortic intramural hematoma: an analysis from the International

Registry of Acute Aortic Dissection. Circulation 2012;126:S91-6.

13. Tittle SL, Lynch RJ, Cole PE, et al. Midterm follow-up of penetrating ulcer and intramural hematoma of the aorta. J Thorac Cardiovasc Surg 2002;123:1051-9.

14. Tanaka A, Leake S, Estrera AL. Management strategies in acute type B aortic intramural hematoma. Curr Opin Cardiol 2017;32:687-91.

15. von Kodolitsch Y, Nienaber CA, Dieckmann C, et al. Chest radiography for the diagnosis of acute aortic syndrome. Am J Med 2004;116:73-7.

16. Kang DH, Song JK, Song MG, et al. Clinical and echocardiographic outcomes of aortic intramural hemorrhage compared with acute aortic dissection. Am J Cardiol 1998;81:202-6.

17. Sundt, TM. Intramural hematoma and penetrating atherosclerotic ulcer of the aorta. (discussion S46-50) Ann Thorac Surg 2007;83:S835-41.

18. Nienaber CA. The role of imaging in acute aortic syndromes. Eur Heart J Cardiovasc Imaging 2013;14:15-23.

19. $\mathrm{Hu} \mathrm{W}$, Schiele $\mathrm{F}$, Meneveau $\mathrm{N}$, et al. The potential value of intravascular ultrasound imaging in diagnosis of aortic intramural hematoma. J Geriatr Cardiol 2011;8:224-9.

20. Trimarchi S, Eagle KA, Nienaber CA, et al. Importance of refractory pain and hypertension in acute type

Cite this article as: $\mathrm{Al}$ Rstum Z, Tanaka A, Eisenberg SB, Estrera AL. Optimal timing of type A intramural hematoma repair. Ann Cardiothorac Surg 2019;8(5):524-530. doi: 10.21037/acs.2019.07.08
B aortic dissection: insights from the International Registry of Acute Aortic Dissection (IRAD). Circulation 2010;122:1283-9.

21. Yamada T, Tada S, Harada J. Aortic dissection without intimal rupture: diagnosis with MR imaging and CT. Radiology 1988;168:347-52.

22. Moizumi Y, Komatsu T, Motoyoshi N, et al. Management of patients with intramural hematoma involving the ascending aorta. J Thorac Cardiovasc Surg 2002;124:918-24.

23. Kan CB, Chang RY, Chang JP. Optimal initial treatment and clinical outcome of type A aortic intramural hematoma: a clinical review. Eur J Cardiothorac Surg 2008;33:1002-6.

24. Sandhu HK, Tanaka A, Charlton-Ouw KM, et al. Outcomes and management of type A intramural hematoma. Ann Cardiothorac Surg 2016;5:317-27.

25. Svensson LG, Adams DH, Bonow RO, et al. Aortic valve and ascending aorta guidelines for management and quality measures: executive summary. Ann Thorac Surg 2013;95:1491-505.

26. Robbins RC, McManus RP, Mitchell RS, et al. Management of patients with intramural hematoma of the thoracic aorta. Circulation 1993;88:II1-10.

27. Matsushita A, Fukui T, Tabata M, et al. Preoperative characteristics and surgical outcomes of acute intramural hematoma involving the ascending aorta: A propensity score-matched analysis. J Thorac Cardiovasc Surg 2016;151:351-8.

28. Moizumi Y, Komatsu T, Motoyoshi N, et al. Clinical features and long-term outcome of type A and type B intramural hematoma of the aorta. J Thorac Cardiovasc Surg 2004;127:421-7. 